\title{
A splicing image detecting metheod based on original image estimation
}

\author{
Yun yi LIANG* Ying WANG \\ * Faculty of Automation of Qingdao University, Qingdao, China
}

\begin{abstract}
The image splicing method is commonly used for forged digital images. This paper proposes a detection method for the splicing image. By the color filter array interpolation, the original image is estimated and the consistency of the adjacent pixels is reconstructed. Use the adjacent pixels consistency of the test image to detect the degree of deviation from the original image, so that the location of the splicing image will be detected. The experiments show that the method can effectively detect the position, size and shape of the splicing image.
\end{abstract}

\section{Keywords-Digital image forensics; Image splicing; CFA}

\section{INTRODUCTION}

In the era of digital image applications, due to the widespread popularity of digital cameras, as well as powerful image processing software (such as Adobe Photoshop), so that anyone can easily produce a digital image forgery. Although they make these false images for various purposes, but there are malicious dissemination of false news and confusions of the situation. Therefore, the blind forensics technology which only based on the digital image data itself has become a hot topic.

In the process of making fake digital image, image splicing is usually the first step. Therefore, image splicing detection and forensics, has became the focus of attention the researchers. On an image of the splicing operation, the literature $[4,5]$ has proposed the quantization discrete cosine transform coefficients and principal component analysis to test the image. Literature [6] proposed a method based on discrete wavelet transform, but can only be detected in the same splicing image. Literature [7, 8] proposes a method based on scale invariant feature transform. Literature [9] also proposed Zernike array forensic methods to detect rotation copy and paste. Based on the different image splicing, literature [10] proposed a model based on the dual-spectrum analysis of the audio signal dual consistency for the splicing image detection, detection correct rate of the detection is about $70 \%$. However, these methods all use the statistical characteristics of the image model to classify the normal image and the splicing image, but can not flag the splicing region. In the actual operation of forensics, it could be a great help for the forensic analyser.

In this paper, by calculating coefficients of the CFA interpolation, the image re-sampling and re-CFA interpolation to get estimation of the original image, then to point out the position of the splicing image. Experiments show that the proposed algorithm can accurately locate the forged splicing image.

\section{GENERAL MODEL OF THE SPLICING IMAGE POSITIONING}

In order to solve the problem of the positioning in the splicing image, and the dependence of the statistical detection method and training sample, this paper presents the original image forensic analysis model. The basic idea of this model is to rebuild the continuity of the original signal and consistency of adjacent pixels damaged by the splicing operation. For the original image that has not been spliced, its estimate of the "original" images and the test images have similar signal continuity. And for the part of the original image signal, due to its destruction in the continuity and consistency of adjacent pixels, the estimate of the original image and the splicing images region are different compare with estimated image. Figure 1 is a general model. The model is composed of three parts. Their respective roles are as follows:

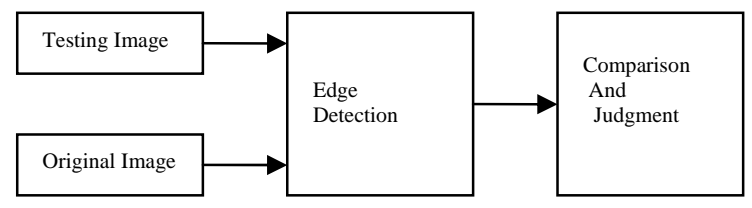

Figure 1: the mode of splicing image positioning

(1) Original estimate: estimate the original image, and reconstruct characteristics of the original image;

(2) Edge Detection: the edge portion of the image is the region which most prone to signal discontinuities;

(3) Comparison and judgment: compare the characteristics between the test image and the estimation of the original image. Then judge the forged region of the image.

\section{ORIGINAL ESTIMATED BASED ON CFA INTERPOLATION}

In the model of the image splicing positioning forensics, the most important step is the original image estimated. The good original image estimation method can rebuilt and restore the original signal statistical characteristics from the splicing image as much as possible, and for the original image it will maintain its original statistical characteristics. CFA interpolation is an important operation in the vast majority of digital camera imaging process, it uses signals' the cyclical and consistency of the image adjacent pixels which is an important reason to cause signal continuity, therefore this paper proposed the use of the CFA interpolation to simulate the image of the imaging process and to rebuild the statistical characteristics of the original signal. The Flow chart as shown in Figure 2 


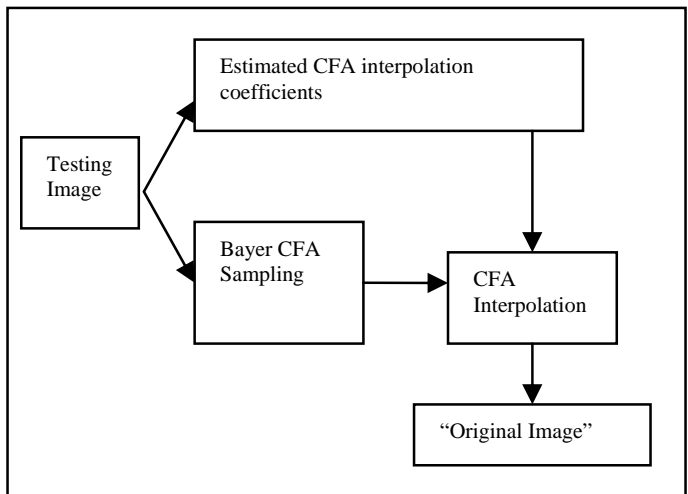

Figure 2: the mode of the CFA interpolation

For reducing the manufacturing cost of the digital camera, as well as consideration of the imaging device production process requirements, the digital camera for one pixel often using CFA only capture one hue of RGB, the pixel point of the other two hue generally calculated by the camera's CFA interpolation algorithm. The most common mode of CFA is the Bayer CFA, as shown in Figure 3. Accordingly, in the image data obtained by the camera, and only $1 / 3$ of the data is directly derived from the sensor imaging, while the remaining $2 / 3$ is set by the $1 / 3$ of the data obtained through CFA interpolation algorithm interpolation. Common CFA interpolation algorithm can be divided into two categories. One category is bilinear and dual cubic interpolation on behalf of the non-adaptive interpolation algorithm, another class based on gradient as the representative of adaptive interpolation algorithm. Imaging process using the CFA interpolation algorithm is not recorded in the digital image, so if we want to take advantage of the CFA interpolation to original image estimated that the first step is the CFA interpolation coefficients to original image.

This paper uses the Bayer CFA as the estimated image mode, the reason is that: on the one hand, taking into account that the Bayer CFA is the most widely used mode; On the other hand, even the images are not estimate by the Bayer CFA style, but the actual sampling point and the adjacent pixels also have inherent correlation. So, Bayer CFA model can also estimate this correlation.

All CFA interpolation algorithms, the basic idea is that use the linear combinations of pixels in the adjacent of the interpolation points to obtain the pixel values of the point, and therefore can use the following linear model to describe it ( $G$ channel interpolation, for example):

$$
y^{g}=\sum_{i=1}^{k} a_{i}^{r} x_{i}^{r}+\sum_{i=1}^{k-1} a_{i}^{g} x_{i}^{g}+\sum_{i=1}^{k} a_{i}^{b} x_{i}^{b}+\delta
$$

wherein $y^{g}$ represents the pixel value of the $G$ channel, $x_{i}^{r}, x_{i}^{g}$ and $x_{i}^{b}$ denote the pixel values of the adjacent of the interpolation points $R, G$ and $B$ channels, $a_{i}^{r}, a_{i}^{g}$ and $a_{i}^{b}$ respectively represent the interpolation coefficients, $\mathrm{k}$ is the adjacent size, $\delta$ is the image noise. All the interpolation points of the $G$ channel pixel written in vector form, in accordance with the manner of the formula (1), then we can get the following formula:

$$
Y^{g}=\sum_{i=1}^{k} a_{i}^{r} X_{i}^{r}+\sum_{i=1}^{k-1} a_{i}^{g} X_{i}^{g}+\sum_{i=1}^{k} a_{i}^{b} X_{i}^{b}+\Delta
$$

In order to estimate the interpolation coefficient, $a_{i}^{r}$, $a_{i}^{g}$ and $a_{i}^{b}$ statistical stability. This article uses the covariance to calculate the formula (2). Covariance has a linear characteristic, and the interference items $\Delta$ are independent with vectors of the pixel value --$\operatorname{cov}\left(\Delta, X_{i}^{r, g, b}\right)=0$. Therefore, we can conclude:

$$
\begin{aligned}
& \operatorname{cov}\left(Y^{g},\right.\left.X_{l}^{r}\right)=\sum_{i=1}^{k} a_{i}^{r} \operatorname{cov}\left(X_{i}^{r}, X_{l}^{r}\right) \\
&+\sum_{i=1}^{k-1} a_{i}^{g} \operatorname{cov}\left(X_{i}^{g}, X_{l}^{r}\right) \\
&+\sum_{i=1}^{k} a_{i}^{b} \operatorname{cov}\left(X_{i}^{b}, X_{l}^{r}\right), l=1, \ldots, k \text { (3) } \\
& \operatorname{cov}\left(Y^{g}, X_{l}^{g}\right)=\sum_{i=1}^{k} a_{i}^{r} \operatorname{cov}\left(X_{i}^{r}, X_{l}^{g}\right) \\
&+\sum_{i=1}^{k-1} a_{i}^{g} \operatorname{cov}\left(X_{i}^{g}, X_{l}^{g}\right) \\
&+\sum_{i=1}^{k} a_{i}^{b} \operatorname{cov}\left(X_{i}^{b}, X_{l}^{g}\right), l=1, \ldots, k-1(4) \\
& \operatorname{cov}\left(Y^{g}, X_{l}^{b}\right)=\sum_{i=1}^{k} a_{i}^{r} \operatorname{cov}\left(X_{i}^{r}, X_{l}^{b}\right) \\
&+\sum_{i=1}^{k-1} a_{i}^{g} \operatorname{cov}\left(X_{i}^{g}, X_{l}^{b}\right) \\
&+\sum_{i=1}^{k} a_{i}^{b} \operatorname{cov}\left(X_{i}^{b}, X_{l}^{b}\right), l=1, \ldots, k(5)
\end{aligned}
$$

According to formula (1) shows, a total of 3k-1 CFA interpolation coefficients are, therefore, according to the formula (3)-(5), we can establish 3k-1 equation. Then we can through the matrix to calculate all interpolation coefficients.

In the basic unit of Bayer CFA $2 \times 2$ use different sampling rate between channel $G$ and $R / B$, in order to guarantee the quality of the image edge, different gradient use different way to interpolate. So we need to classify the pixel point, and constructed in accordance with the different categories of the linear interpolation model, and then estimate the interpolation coefficients.

First, consider the classification of the different sampling points of the basic structure of Figure 3 in the Bayer CFA. In this paper, we make each $G$ component as one class, $B / R$ as one class of each component, and the RIB component of $G 1$ and $G 2$ as two categories. It means that the eight hue component missing in the $2 \times 2$ unit, six of 
them separately build its own mode (except the two $G$ component of the RGB sampling point): $R_{G 1}, R_{G 2}, R_{B}$, $B_{G 1}, B_{G 2}, B_{R}$.

Further, for the two $G$ component of the $R / B$ sampling point interpolation model $G$, taking into account the difference of the gradient of $G$ channel pixel point, the interpolation method mostly are different. Reference [16] method, the $G_{R / B}$ is divided into horizontal, vertical and diagonal direction interpolation points, that is $G_{R / B-H}$, $G_{R / B-V}$ and $G_{R / B-D}$. Gradient calculating [16] and classifications, such as formula (6)--(8) below, wherein, $P_{x, y}$ indicates the sampling point of Bayer CFA after sampling the $\mathrm{R} / \mathrm{B}$ pixel values .

$$
\left.\begin{array}{c}
H_{x, y}=\left|P_{x, y-2}+P_{x, y+2}-2 P_{x, y}\right| \\
V_{x, y}=\left|P_{x, y-2}+P_{x, y+2}-2 P_{x, y}\right| \\
\text { HPoint, } H_{x, y}<V_{x, y} \\
\text { VPoint, } H_{x, y}>V_{x, y} \\
\text { DPoint, } H_{x, y}=V_{x, y}
\end{array}\right\}
$$

Thus it can be obtained, all pixels to be estimated, is divided into 9 categories: $G_{R / B-H}, G_{R / B-V}, G_{R / B-D}, R_{G 1}$, $R_{G 2}, R_{B}, B_{G 1}, B_{G 2}$ and $B_{R}$. And solving each of its equations build in accordance with the formula (3) -- (5) can get the estimated the CFA interpolation coefficients. Then sample the testing image by CFA interpolation after getting the CFA interpolation coefficients. Missing hue component of each sampling point, corresponds to one pixel in the above classification method, the estimated the CFA interpolation coefficient the obtained in accordance with the corresponding class, re CFA interpolation of the missing hue components, to thereby obtain the estimated "original" images.

\section{Positioning THE LOCATION OF THE IMAGE SPLICING}

When we get the original image, we can obtain the location of the splicing image by testing the continuity and consistency of the image edge point.

Since splicing forged main purpose is to introduce a new object, the edge point will inevitably exist, through the edge detection can improve detection efficiency, and by means of edge detection algorithms to improve the positioning accuracy of the splicing forged. This article is use "Canny edge detection", it has a good performance in the noise suppression and edge detection.

In order to compare the signal continuity of the testing image $I$ and original image $I^{\text {est }}$ at the edge point (x, y), we need to quantitatively describe the continuity of the adjacent. This paper use following characteristics to measure:

$$
\begin{aligned}
& d I_{x, y}=\max \left(I_{x, y}^{K}-\frac{I_{x-1, y}^{K}+I_{x+1, y}^{K}}{2},\right. \\
& \left.I_{x, y}^{K}-\frac{I_{x, y-1}^{K}+I_{x, y+1}^{K}, I_{x, y}^{K}-\frac{I_{x-1, y+1}^{K}+I_{x+1, y-1}^{K}}{2},(9)}{2}\right) \\
& \left.I_{x, y}^{K}-\frac{I_{x-1, y-1}^{K}+I_{x+1, y+1}^{K}}{2}\right)
\end{aligned}
$$

Wherein IK indicates the pixel value of the edge point, $K \in\{R, G, B\}$. The formula (9) describes the maximum deviation of hue channel pixel values and its row-direction, column-direction and two the diagonal direction of adjacent pixels. The formula (10) gets the maximum deviation of all hue channels of the pixels adjacent in the four directions.

$$
d I_{x, y}=\max \left(d I_{x, y}^{K}\right), K \in\{R, G, B\}
$$

We can use the same way to obtain the $d I_{x, y}^{e s t}$. It means that we can get the maximum deviation of all hue in 4 channels about the adjacent pixel of the original image. $d=\max \left(d I_{x, y}-d I_{x, y}^{\text {est }}\right)$

Reflect the differences of characteristics between the testing image and the estimated original image. Therefore, it used for the judgment whether the pixel is splicing pixels:

$$
\left.\begin{array}{l}
I_{x, y} \in \phi_{F}, d \geq T \\
I_{x, y} \in \phi_{A}, d<T
\end{array}\right\}
$$

Here $\phi_{F}$ and $\phi_{A}$ is the splicing pixels and original pixels respectively. $T$ is a decision threshold, there are 4821 of the original image captured by 22 different models of digital cameras and count these $d$ value. The edge points of the original image was shown as standards unilateral normal distribution, but the spliced image's edge points obedience the general unilateral normal distribution $N\left(\mu, \sigma^{2}\right)$, which $\mu$ and $\sigma$, respectively represent the distribution of the mean and standard deviation. Therefore, in order to distinguish the two characteristic values. This paper selected the second threshold value as the standards. And they respectively are the balance false alarm rate and undetected rate about the minimum misclassification standard, and the standard of considering the judgment of the original image edge points correctly confidence interval:

$$
\begin{aligned}
& T 1=\arg \min \left(p\left(d_{a}>T 1\right)+p\left(d_{s}<T 1\right)\right) \\
& T 2=\min (T), \text { s.t. } \sum_{i=1}^{T} p_{a}^{i}>0.99
\end{aligned}
$$

Formula (12) represents that choose the threshold which makes the sum of fault points of the original image edge point and splicing edge point minimum, Wherein $p\left(d_{a}>T 1\right)$ represents the original image misclassification 
probability, $p\left(d_{s}<T 1\right)$ indicates the probability of the spliced edge points. Formula (13) indicates the minimum threshold where the value of the correct degree of confidence more than 0.99 about the original image.

\section{EXPERIMENTAL RESULTS}
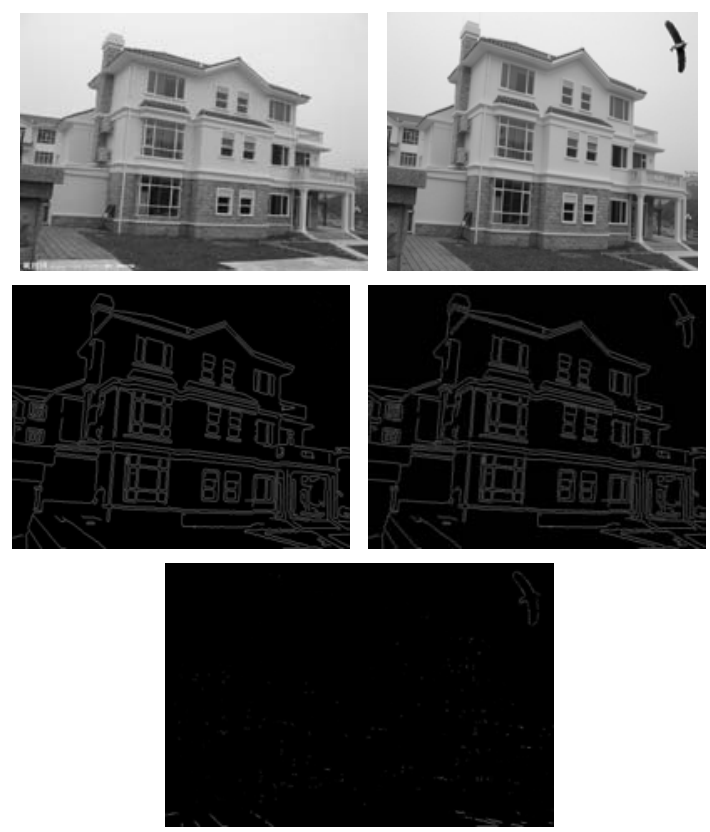

VI. CONCLUSIONS

For accurate positioning of the forgery area is a very challenging task. This paper presents a method to locate the spliced region. Through the CFA interpolation coefficient estimation, the testing image is to be re-interpolation, and get the estimation of the testing image. Take advantage of the original estimation to compare the continuity and consistency of the neighboring pixels and the edge point, and then to judge a splicing forgery edge points. The experiment shows that the method can accurately detect and locate the image stitching forged region.

\section{ACKNOWLEDGEMENTS}

\section{National Natural Science Foundation (60973048)}

\section{REFERENCES}

[1] Malhdian B and Saic S. A bibliography on blind methods for identifying image forgery [J]. Singnal Processing: Image Communication, 2010,25(6): 389-399.

[2] Christlcin V. Riese C. and Angelopoulou E. A study on features for the detection of copy-move forgeries [C]. Information Security Solutions Europe, Belin, Germany, Oct. 5-7, 2010: 1-12.

[3] Shivakmnar B and Sathosh S. Detecting copy-move forgery in digital images: a survey and analysis of current methods [J]. Global Journal of Computer Science and technology, 2011,10(7): 61-65.

[4] Fridrich J, Soukal D, and Lukas J. Detection of copy-move forgery in digital images [C]. Digital Forensics Research Workshop, Cleveland, USA, Aug. 6-8,2003:1-10.
[5] Popesen A C and Farid H. Exposing digital forgeries by detecting duplicated image regions http://www.cs.dartmounth. edu/farid/dfd/mdex. php/attachments/single/2,2,2004.

[6] Khan S and Kulkarni A. An efficient method for detection of copymove forgery using discrete wavelet transform [J]. International Journal on Computer Science and Engineering, 2010,2(5): 1801-1806.

[7] Pan Xun-yu and Lyu Si-wei. Detecting image region duplication using SIFT features [C]. International Conference on Acoustics, Speech, Signal Processing, Dallas, USA. Mar.14-19,2010: 1702-1705.

[8] Amerini I, Ballan L, Caldelli R, et al. Geometric tampering estimation by means of a SIFT-based forensic analysis [C]. International Conference on Acoustics, Speech, and Signal Processing,Dallas, USA, Mar. 14-19, 2010: 1702-1705.

[9] Ryu Seung-Jin, Lee Min-Jeong, and Lee Heung-kyu. Detection of copy-rotate-move forgery using zernike moments [C]. International Workshop on Information Hiding, Calgary, Cannadam June 28-30, 2010:5165.

[10] Ng T T and Chang S F. A model for image splicing [C]. IEEE International Conference on Image Processing, Singapore, Oct. 24-27, 2004: 1169-1172.

[11] Ng T T , Chang S F and sun Q. Blind detetion of photomontage using higher order statisties [C].IEEE International Symposium on Circuits and Systems, Vancouver,Canada,May 23-26,2004: 688-691.

[12] Hsu Y F and Chang S F. Detecting image splicingusing geometry invariants and camera characteristicsconsistency[C].IEEE International Conference Multimedia \& Expo,Toronto, Canada, July 9-12,2006:549-552.

[13] Freeman W T. Median filter for reconstructing missing color samples[P]. Patent, USA, 4642678,1988. 